\title{
Clinical pharmacokinetics and pharmacodynamics of ivosidenib, an oral, targeted inhibitor of mutant IDH1, in patients with advanced solid tumors
}

\author{
Bin Fan ${ }^{1} \cdot$ Ingo K. Mellinghoff ${ }^{2} \cdot$ Patrick Y. Wen $^{3} \cdot$ Maeve A. Lowery $^{4} \cdot$ Lipika Goyal $^{5} \cdot$ William D. Tap $^{6,7}$. \\ Shuchi S. Pandya ${ }^{8}$ - Erika Manyak ${ }^{1} \cdot$ Liewen Jiang $^{9} \cdot$ Guowen Liu $^{1} \cdot$ Tara Nimkar $^{10} \cdot$ Camelia Gliser $^{8} \cdot$ Molly Prahl \\ Judge $^{8} \cdot$ Sam Agresta ${ }^{8} \cdot$ Hua Yang ${ }^{1} \cdot$ David Dai $^{1}$
}

Received: 13 February 2019 / Accepted: 28 March 2019 / Published online: 26 April 2019

(C) The Author(s) 2019

\begin{abstract}
Summary
Background Mutant isocitrate dehydrogenase 1 and 2 (IDH1/IDH2) enzymes produce the oncometabolite D-2-hydroxyglutarate (2-HG). Ivosidenib (AG-120) is a targeted mutant IDH1 inhibitor under evaluation in a phase 1 dose escalation and expansion study of IDH1-mutant advanced solid tumors including cholangiocarcinoma, chondrosarcoma, and glioma. We explored the pharmacokinetic (PK) and pharmacodynamic (PD) profiles of ivosidenib in these populations. Methods Ivosidenib was administered orally once (QD) or twice (BID) daily in continuous 28-day cycles; 168 patients received $\geq 1$ dose within the range $100 \mathrm{mg}$ BID to $1200 \mathrm{mg}$ QD. PK and PD were assessed using validated liquid chromatography-tandem mass spectrometry assays. Results Ivosidenib demonstrated good oral exposure after single and multiple doses, was rapidly absorbed, and had a long terminal half-life (mean 40-102 h after single dose). Exposure increased less than dose proportionally. Steady state was reached by day 15 , with moderate accumulation across all tumors (1.5- to 1.7-fold for area-under-the-curve at $500 \mathrm{mg} \mathrm{QD}$ ). None of the intrinsic and extrinsic factors assessed affected ivosidenib exposure, including patient/disease characteristics and concomitant administration of weak CYP3A4 inhibitors/inducers. After multiple doses in patients with cholangiocarcinoma or chondrosarcoma, plasma 2-HG was reduced by up to $98 \%$, to levels seen in healthy subjects. Exposure-response relationships for safety and efficacy outcomes were flat across the doses tested. Conclusions Ivosidenib demonstrated good oral exposure and a long half-life. Robust, persistent plasma 2-HG inhibition was observed in IDH1-mutant cholangiocarcinoma and chondrosarcoma. Ivosidenib $500 \mathrm{mg}$ QD is an appropriate dose irrespective of various intrinsic and extrinsic factors. Trial Registration ClinicalTrials.gov (NCT02073994).
\end{abstract}

Keywords 2-hydroxyglutarate $\cdot$ Isocitrate dehydrogenase 1 inhibitor $\cdot$ Ivosidenib $\cdot$ Pharmacodynamics $\cdot$ Pharmacokinetics

\section{Introduction}

Isocitrate dehydrogenase (IDH) is a critical metabolic enzyme, catalyzing the oxidative decarboxylation of isocitrate to pro-

Erika Manyak, Sam Agresta, and David Dai Affiliation at time of study

Electronic supplementary material The online version of this article (https://doi.org/10.1007/s10637-019-00771-x) contains supplementary material, which is available to authorized users.

Bin Fan

bin.fan@agios.com

Extended author information available on the last page of the article duce carbon dioxide and alpha-ketoglutarate $(\alpha-\mathrm{KG})$. Mutations in the $I D H 1$ and $I D H 2$ genes are found in multiple hematologic and solid tumors, including acute myeloid leukemia (AML) and glioma. Mutant IDH enzymes are not catalytically inactive, but rather possess a novel enzymatic activity, catalyzing the reduction of $\alpha-\mathrm{KG}$ to the oncometabolite D-2hydroxyglutarate $(2-\mathrm{HG})[1,2]$. In normal cells, $2-\mathrm{HG}$ is present at low levels. However, in cells with IDH1/IDH2 mutant enzymes, the accumulation of 2-HG alters a number of downstream cellular activities, causing epigenetic dysregulation and consequently a block in cellular differentiation, leading to tumorigenesis [3-5].

Ivosidenib (AG-120) is a selective, potent inhibitor of the mutant IDH1 protein [6]. Preclinical studies showed that 
treatment with ivosidenib decreased intracellular 2-HG levels in IDH1-mutant AML cells in vitro [7], and resulted in 2-HG inhibition in tumors in an IDH1-mutant xenograft mouse model [6]. These data were used to predict the exposure required for efficacy in humans. The inhibition of $2-\mathrm{HG}$ production by ivosidenib translated well from preclinical models to humans [8]. In a phase 1 study, ivosidenib $500 \mathrm{mg}$ once daily (QD) was shown to have an acceptable safety profile, and was associated with durable remissions in patients with advanced hematologic malignancies, including relapsed/refractory (R/R) AML and myelodysplastic syndrome [9]. On the basis of data from that study, ivosidenib received United States Food and Drug Administration (FDA) approval for the treatment of adult patients with R/R AML with a susceptible IDH1 mutation as detected by an FDA-approved test [10].

Ivosidenib is also being investigated in an ongoing phase 1 study that enrolled patients with advanced solid tumors [11-14]. The safety and efficacy data from this study are reported in separate publications (manuscripts in preparation). Here we report the pharmacokinetic (PK) and pharmacodynamic (PD) data associated with ivosidenib treatment in these patients, and the effects of intrinsic and extrinsic factors on ivosidenib clearance.

\section{Methods}

\section{Study design and treatment}

This was a phase 1 , multicenter, open-label, dose escalation and expansion study (clinicaltrials.gov number NCT02073994). The primary objective was to assess the safety and tolerability of ivosidenib in patients with advanced solid tumors harboring an IDH1 mutation. Secondary objectives included the characterization of ivosidenib PK and the PK/PD relationship of ivosidenib and 2-HG.

The study was conducted in accordance with the principles of the Declaration of Helsinki and Good Clinical Practice guidelines and was approved by the appropriate review boards at participating sites. Written informed consent was obtained from all patients.

In the dose escalation portion, patients with 1) glioma and 2) non-glioma solid tumors were enrolled into sequential cohorts using a standard $3+3$ design. Patients with glioma received $100 \mathrm{mg}$ twice daily (BID), or $300,500,600$, or $900 \mathrm{mg}$ QD ivosidenib in continuous 28-day cycles. Patients with cholangiocarcinoma, chondrosarcoma, and other solid tumors received ivosidenib $100 \mathrm{mg} \mathrm{BID}$, or $300,400,500,800$, or $1200 \mathrm{mg}$ QD in continuous 28-day cycles. At least 3 patients in each cohort also received a single dose 3 days prior to the start of multiple dosing (i.e., day -3). Patients in the expansion portion all received $500 \mathrm{mg}$ QD ivosidenib in continuous 28day cycles.

\section{Patients}

All patients were required to be at least 18 years of age, and have an advanced solid tumor with an $I D H 1$ mutation, with an expected survival of at least 3 months, and adequate bone marrow, hepatic, and renal function. Other key inclusion criteria for dose escalation included histologically or cytologically confirmed advanced solid tumors that had recurred or progressed following standard therapy, and evaluable disease by Response Assessment in Neuro-Oncology (RANO) criteria for patients with glioma, or by Response Evaluation Criteria in Solid Tumors (RECIST) v1.1 for patients with other solid tumors. For expansion, patients with cholangiocarcinoma had to have a stage II, III, or IV intra-hepatic, extrahepatic, or perihilar tumor that was not amenable to curative resection, transplantation, or ablative therapies (tumors of mixed histology were not allowed), and must have progressed following a gemcitabine-based regimen; patients with chondrosarcoma had to have a tumor that was either locally advanced or metastatic and not amenable to complete surgical excision (any subtype was permitted); patients with nonenhancing glioma had to have a tumor that had progressed within 12 months or less and was solely non-enhancing on magnetic resonance imaging (MRI), and have had no prior surgery or radiation therapy within 6 months of enrollment. Patients with other solid tumors in the expansion portion had to have a tumor that was refractory to conventional therapy or be unable to tolerate conventional therapy.

Key exclusion criteria for all patients included systemic anticancer therapy or radiotherapy less than 21 days prior to the first ivosidenib dose, use of investigational agents less than 14 days (or 5 half-lives) prior to the first dose, and concomitant use of sensitive substrates of cytochrome P450 (CYP) 3A4, or P-glycoprotein (P-gp).

\section{PK and PD assessments}

For the first 3 patients enrolled in each cohort in the dose escalation portion, blood samples for assessment of plasma concentrations of ivosidenib and 2-HG were collected up to $72 \mathrm{~h}$ postdose on day -3 . These samples were optional for any further patients enrolled in these cohorts. Further blood samples were collected from all patients up to $10 \mathrm{~h}$ postdose on cycle 1 , day 15 and cycle 2 , day 1 . A subset of samples was also used for evaluation of plasma $4 \beta$-hydroxycholesterol $(4 \beta-\mathrm{OHC})$ and $4 \beta-\mathrm{OHC}$ :cholesterol ratios. Blood samples for assessment of 2-HG only were also collected at screening.

In the expansion portion, blood samples for assessment of plasma concentrations of ivosidenib and 2-HG were collected from all patients predose, and at 2, 3, 4, 6, and $8 \mathrm{~h}$ ( $\pm 10 \mathrm{~min})$ 
postdose on cycle 1 , day 1 and cycle 2 , day 1 . Additional predose samples were taken on cycle 1 , day 8 ; cycle 1 , day 15 ; cycle 3 , day 1 ; and end of treatment.

Tumor biopsies were also collected for 2-HG assessment at screening, and at day 1 of cycle 3. Further biopsies were collected depending on the status of disease at cycle 7, day 1 or at any time that disease progression was suspected and/or at the end of treatment.

Plasma ivosidenib was measured using two validated liquid chromatography-tandem mass spectrometry (LC-MS/MS) methods. The lower limit of quantification (LLOQ) was $1.00 \mathrm{ng} / \mathrm{mL}$ (low-range assay) or $50.0 \mathrm{ng} / \mathrm{mL}$ (high-range assay). The two methods were cross-validated and found to deliver comparable results with acceptable limits. Plasma 2HG concentrations were measured using a qualified LC-MS/ MS method with an LLOQ of $30.0 \mathrm{ng} / \mathrm{mL}$. Concentrations of 2-HG in tumor biopsy samples were quantified using qualified LC-MS/MS methods with an LLOQ of $7.5 \mu \mathrm{g} / \mathrm{g}$ for brain tumor homogenate, $100 \mathrm{ng} / \mathrm{g}$ for liver tumor homogenate (low-range assay), and $30 \mu \mathrm{g} / \mathrm{g}$ for liver tumor homogenate (high-range assay). Plasma $4 \beta-\mathrm{OHC}$ and cholesterol were measured using a validated LC-MS/MS method. The LLOQ was $5 \mathrm{ng} / \mathrm{mL}$ for $4 \beta-\mathrm{OHC}$ and $25 \mu \mathrm{g} / \mathrm{mL}$ for cholesterol in human plasma.

Ivosidenib plasma PK parameters and 2-HG PD parameters were calculated using non-compartmental methods (Phoenix ${ }^{\circledR}$ WinNonlin $^{\circledR}$ 6.3; Certara, Princeton, NJ). PK parameters included area under the plasma concentration-time curve (AUC), maximum plasma concentration $\left(\mathrm{C}_{\max }\right)$, time to maximum plasma concentration $\left(\mathrm{T}_{\max }\right)$, terminal elimination half-life $\left(\mathrm{t}_{1 / 2}\right)$, and apparent oral clearance at steady state $\left(\mathrm{CL}_{\mathrm{ss}} / \mathrm{F}\right)$. Accumulation ratio $\left(\mathrm{R}_{\mathrm{acc}}\right)$, based on $\mathrm{C}_{\max }$ and AUC, was also assessed during the dose escalation portion. PD (2-HG) parameters included baseline-effect value, area under the effect concentration-time curve $\left(\mathrm{AUEC}_{0-10 \mathrm{~h}}\right.$ for the dose escalation portion, or $\mathrm{AUEC}_{0-8 \mathrm{~h}}$ for the dose escalation and expansion portions), percent change from baseline in AUEC (\% BAUEC $_{0-10 \mathrm{~h}}$ for the escalation portion, or $\% \mathrm{BAUEC}_{0-8 \mathrm{~h}}$ for the dose escalation and expansion portions), average plasma concentration $\left(\mathrm{C}_{\mathrm{avg}}\right)$, and percent inhibition for $\mathrm{C}_{\mathrm{avg}}\left(\% \mathrm{BC}_{\mathrm{avg}}\right)$. For the dose escalation portion, the timematched ratios of $4 \beta-\mathrm{OHC}$ to cholesterol were calculated. Box plots of 2-HG AUEC ${ }_{0-8 \mathrm{~h}}$ and $\mathrm{C}_{\mathrm{avg}}$ versus three categories of ivosidenib dose level $(<500 \mathrm{mg}, 500 \mathrm{mg}$ and $>500 \mathrm{mg}$ ) were plotted at each timepoint to assess trends in the dose range studied (for dose escalation and expansion combined).

\section{Assessment of dose proportionality}

Dose proportionality of ivosidenib $\mathrm{AUC}$ and $\mathrm{C}_{\max }$ at day -3 , and at cycle 1 , day 15 , and cycle 2, day 1 was assessed using a power model over the dose range studied (100 mg BID, and $300 \mathrm{mg}$ to $1200 \mathrm{mg}$ QD). Dose proportionality after multiple dosing was tested using data from the QD dose regimens without the $100 \mathrm{mg}$ BID dose group. To assess dose proportionality, the confidence interval (CI) for the slope was estimated, with each natural logarithm (ln)-transformed PK parameter as the dependent variable and the $\ln$-transformed dose as the fixed effect. Analysis was performed using PK parameter estimates at day -3 , cycle 1 , day 15 , and cycle 2 , day 1 . Dose proportionality was concluded if the $95 \% \mathrm{CI}$ around the slope included 1 and if the slope was between 0.8 and 1.2 (inclusive).

\section{Assessment of effects of intrinsic and extrinsic factors on ivosidenib PK}

Patient characteristics at baseline were used to perform exploratory analyses of intrinsic and extrinsic factors that potentially influenced ivosidenib PK for two subgroups of tumor types: 1) non-glioma (cholangiocarcinoma, chondrosarcoma, and other solid tumors), and 2) glioma (non-enhancing and enhancing). Continuous factors assessed included age, body weight, body mass index, baseline creatinine clearance $\left(\mathrm{CL}_{\mathrm{cr}}\right.$ ), baseline estimated glomerular filtration rate (eGFR), and markers of hepatic function (alanine aminotransferase [ALT], alkaline phosphatase [ALP], aspartate aminotransferase [AST], bilirubin, total protein, and albumin). $\mathrm{CL}_{\mathrm{cr}}$ was calculated using the Cockcroft and Gault equation and eGFR was calculated using the Modification of Diet in Renal Disease - National Kidney Disease Education Program equations [15]. Categorical factors assessed included sex, ethnicity, race, disease status, concomitant CYP3A4 inhibitor and inducer status, baseline hepatic and renal function category, and tumor type. Renal function was categorized as normal $\left(\mathrm{CL}_{\mathrm{cr}} \geq 90 \mathrm{~mL} / \mathrm{min}\right.$ or $\left.\mathrm{eGFR} \geq 90 \mathrm{~mL} / \mathrm{min} / 1.73 \mathrm{~m}^{2}\right)$, mild impairment $\left(\mathrm{CL}_{\mathrm{cr}} \geq 60-89 \mathrm{~mL} / \mathrm{min}\right.$ or eGFR $\geq 60$ $\left.89 \mathrm{~mL} / \mathrm{min} / 1.73 \mathrm{~m}^{2}\right)$, moderate impairment $\left(\mathrm{CL}_{\mathrm{cr}} \geq 30\right.$ $59 \mathrm{~mL} / \mathrm{min}$ or eGFR $\geq 30-59 \mathrm{~mL} / \mathrm{min} / 1.73 \mathrm{~m}^{2}$ ), and severe impairment $\left(\mathrm{CL}_{\mathrm{cr}} \geq 15-29 \mathrm{~mL} / \mathrm{min}\right.$ or eGFR $\geq 15-29 \mathrm{~mL} /$ $\min / 1.73 \mathrm{~m}^{2}$ ). Hepatic function category was based on National Cancer Institute (NCI) organ dysfunction working group $(\mathrm{ODWG})$ criteria for hepatic dysfunction $(0=$ normal, $1=$ mild, $2=$ moderate, $3=$ severe, $4=$ liver transplant) [16].

\section{Exploratory dose/exposure-response analysis}

A total of 6 adverse event categories, based on both laboratory abnormalities and reported adverse events, were included in the exposure-response analyses. Selected adverse events were identified based on safety signals observed within the ivosidenib clinical development program: grade $\geq 3$ adverse events, serious adverse events, grade $\geq 2$ gastrointestinal events (i.e., nausea/vomiting/diarrhea), hepatic enzyme elevations (i.e., newly occurring or worsening laboratory abnormalities of all grades, and grade $\geq 2$ elevations in ALT, AST, or 
bilirubin), grade $\geq 2$ decreases in lymphocytes, and grade $\geq 3$ increases in ALP. The efficacy endpoint of interest was best response. The exposure-response analyses used modeled nominal steady-state $\mathrm{C}_{\max }\left(\mathrm{C}_{\max , \mathrm{ss}}\right)$ or $\mathrm{AUC}\left(\mathrm{AUC}_{\mathrm{ss}}\right)$ as predictors for safety and efficacy variables in the two subgroups of tumor types described earlier. The association between ivosidenib exposure and the selected endpoints was demonstrated by 1) exposure versus adverse event or clinical best response, with vertical boxplots showing exposure distributions, and 2) adverse event probability versus exposure plots showing the event probability for exposure quartiles and logistic regression fit based on the individual data.

\section{Results}

\section{Patient disposition and demographics}

The first patient was enrolled on March 14, 2014 and the study was ongoing at the time of writing. The data cutoff date for the analyses presented here was May 12, 2017. A total of 168 patients received at least 1 dose of ivosidenib, of whom 60 were treated in the dose escalation portion and 108 were treated in the expansion portion (Supplementary Fig. S1). The majority of patients were female (52.4\%), white (78.6\%), and $<60$ years of age $(68.5 \%)$. Tumor types included cholangiocarcinoma (43.5\%), chondrosarcoma (12.5\%), enhancing glioma (18.5\%), non-enhancing glioma (20.8\%), and other solid tumors $(4.8 \%)$ (Supplementary Table S1). Renal function (based on $\mathrm{CL}_{\mathrm{cr}}$ ) was classified as normal in $70.2 \%$ of patients, mild to moderate impairment in $29.2 \%$, and severe impairment in $0.6 \%$. Hepatic function (based on NCI-ODWG Classification) was classed as normal in $71.7 \%$ of patients, mild impairment in $26.4 \%$ and moderate impairment in $1.9 \%$. Patients in the glioma cohort were generally younger than those in the other solid tumor subgroup, with a median age of 41 years (600 mg QD) and 35.5 years (900 mg QD) compared with 57 years (400 mg QD), 61 years ( $800 \mathrm{mg}$ QD), and 55 years (1200 mg QD), respectively. PK and PD were assessed in all 168 patients treated with ivosidenib.

\section{PK}

The single- and multiple-dose PK of ivosidenib are presented for patients with glioma (enhancing and non-enhancing) and non-glioma (cholangiocarcinoma, chondrosarcoma, and other solid tumors).

After a single dose on day -3 , ivosidenib was rapidly absorbed, with median $\mathrm{T}_{\max }$ ranging from approximately 2 to $6 \mathrm{~h}$ across all tumor types (Table 1). After reaching $\mathrm{C}_{\max }$, mean ivosidenib concentrations declined in a bi-exponential manner over the 72 -h postdose sampling period (Supplementary Fig. S2). The exposure of ivosidenib $\left(\mathrm{C}_{\max }\right.$ and AUC) generally increased in a less than dose-proportional manner after a single dose ranging from 100 to $1200 \mathrm{mg}$. At higher dose levels (500, 800, and $900 \mathrm{mg} \mathrm{QD})$, mean $\mathrm{AUC}_{0-}$ ${ }_{72} \mathrm{~h}$ and $\mathrm{C}_{\max }$ in the subgroup of patients with glioma were lower than in the subgroup of patients with non-glioma solid tumors. Interpatient variability was low to moderately high for $\mathrm{C}_{\max }$ and AUC parameters (i.e., geometric coefficient of variation [CV\%] ranged from $7 \%$ to $66.9 \%$ where $n \geq 3$ ). Mean $t_{1 / 2}$ appeared to be shorter for the subgroup of patients with glioma (range $\sim 40-56 \mathrm{~h}$ ), versus the subgroup of patients with non-glioma solid tumors (range $\sim 62-102 \mathrm{~h}$ ).

After multiple doses, ivosidenib was rapidly absorbed, and median $\mathrm{T}_{\max }$ ranges $(\sim 2-3 \mathrm{~h})$ were comparable in patients with glioma and patients with non-glioma solid tumors (Supplementary Tables S2 and S3). Mean concentration-time profiles of ivosidenib on cycle 1 , day 15 were comparable with those on cycle 2, day 1 with QD dosing (data not shown), indicating that steady-state was reached within the first cycle. Exposure of ivosidenib $\left(\mathrm{C}_{\max }\right.$ and AUC) increased in a less than dose-proportional manner at doses from 300 to $1200 \mathrm{mg}$ QD. At the $500 \mathrm{mg}$ QD dose level, area under the curve over the dosing interval $\left(\mathrm{AUC}_{0 \text {-tau }}\right)$ and $\mathrm{C}_{\max }$ in patients with glioma were lower than in patients with non-glioma solid tumors.

Mean $\mathrm{CL}_{\mathrm{ss}} / \mathrm{F}$ generally increased with increasing dose and was slightly higher for patients with glioma (range 6-14 L/h) compared with patients with non-glioma solid tumors (range $3-10 \mathrm{~L} / \mathrm{h}$ ). Ivosidenib showed a consistent trend of moderate accumulation (1.5-fold to 1.7-fold for AUC at $500 \mathrm{mg}$ QD) across tumor types (Supplementary Tables S2 and S3).

After multiple administrations of ivosidenib across the dose range from $100 \mathrm{mg}$ BID and $300 \mathrm{mg}$ QD to $1200 \mathrm{mg}$ QD, mean plasma $4 \beta-\mathrm{OHC}$ :cholesterol ratios showed increases in the range of $86 \%$ to $258 \%$ from baseline to cycle 1 , day 15 or cycle 2 , day 1 , and the increases did not appear to be dose dependent.

\section{Dose proportionality}

The point estimates and $95 \% \mathrm{CI}$ for slope terms of the power models of $\mathrm{AUC}_{0-72 \mathrm{~h}}$ and $\mathrm{C}_{\max }$ after a single dose of ivosidenib on day -3 were 0.455 (95\% CI $0.210-0.700)$ and 0.440 (95\% CI $0.233-0.647)$, respectively, for glioma, and 0.726 (95\% CI $0.513-0.938$ ) and 0.625 (95\% CI 0.419-0.831), respectively, for non-glioma solid tumors. Since the $95 \%$ CIs did not include 1 for any of the PK parameters, dose proportionality was not demonstrated over the dose ranges studied. Overall, the slope was less than 1 , suggesting that $\mathrm{C}_{\max }$ and $\mathrm{AUC}_{0-72 \mathrm{~h}}$ exhibited less than dose-proportional increases in ivosidenib exposure in patients with glioma over the dose range of $100 \mathrm{mg}$ to $900 \mathrm{mg}$, and in patients with non-glioma solid tumors over the dose range of $100 \mathrm{mg}$ to $1200 \mathrm{mg}$ (Fig. 1).

After multiple administrations of ivosidenib, at cycle 2, day 1 , the point estimates and $95 \% \mathrm{CI}$ for slope terms of the power 
models for $\mathrm{AUC}_{0 \text {-tau }}$ and $\mathrm{C}_{\max }$ were $0.249(95 \% \mathrm{CI}-0.109$ 0.606 ) and 0.227 (95\% CI $-0.179-0.634)$, respectively, for glioma, and 0.402 (95\% CI $0.038-0.767)$ and 0.458 (95\% CI 0.151-0.764), respectively, for non-glioma solid tumors. As described for the single-dose analysis above, the $95 \%$ CIs did not include 1 for any of the PK parameters, and the slope was less than 1 , suggesting that $\mathrm{C}_{\max }$ and $\mathrm{AUC}_{0 \text {-tau }}$ at steady state exhibited less than dose-proportional increases in ivosidenib exposure in patients with glioma over the dose range of $300 \mathrm{mg}$ to $900 \mathrm{mg}$, and in patients with non-glioma solid tumors over the dose range of $300 \mathrm{mg}$ to $1200 \mathrm{mg}$.

\section{Effect of intrinsic and extrinsic factors on ivosidenib PK}

Exploratory graphical analysis suggested that there was no trend between ivosidenib PK parameters and the continuous baseline characteristics of age, body weight, body mass index, markers of hepatic and renal function, use of CYP3A inducers/inhibitors, albumin, sex, ethnicity, race, tumor type, and performance status (data not shown).

Exploratory assessments of categorical factors demonstrated that there was no apparent effect of mild or moderate renal impairment or mild hepatic impairment (Fig. 2a-b), or concomitant administration of weak CYP3A4 inhibitors or inducers (Fig. 2c-d) on ivosidenib $\mathrm{CL}_{\mathrm{ss}} / \mathrm{F}$. However, given the small sample size of patients with moderate hepatic impairment in the non-glioma solid tumor subgroup, the results of the comparison between moderate hepatic impairment and normal hepatic function should be interpreted with caution.

\section{Pharmacodynamics}

In patients with glioma (non-enhancing and enhancing), mean plasma 2-HG baseline concentrations ranged from $49.7 \mathrm{ng} /$ $\mathrm{mL}$ to $97.1 \mathrm{ng} / \mathrm{mL}$ and were similar to 2-HG levels previously observed in healthy subjects $(72.6 \pm 21.8 \mathrm{ng} / \mathrm{mL}$; Agios data on file). Mean plasma 2-HG concentrations after ivosidenib treatment in patients with glioma remained similar to those observed in healthy subjects, therefore decreases from baseline in plasma 2-HG concentrations were not calculated, and this patient population was not investigated further with regard to PD parameters.

In patients with cholangiocarcinoma and chondrosarcoma, mean plasma 2-HG concentrations were elevated at baseline (ranging from $222 \mathrm{ng} / \mathrm{mL}$ to $5220 \mathrm{ng} / \mathrm{mL}$ and from $94 \mathrm{ng} / \mathrm{mL}$ to $1490 \mathrm{ng} / \mathrm{mL}$, respectively) compared with healthy subjects. After one week of continuous ivosidenib dosing, plasma 2HG was inhibited by up to $98 \%$ compared with baseline, to levels consistent with those seen in healthy subjects, and this decrease was maintained through the treatment period (up to 17 cycles of dosing). 
Fig. 1 Representative graph of dose proportionality assessment for $\mathrm{AUC}_{0-72 \mathrm{~h}}$ (a) and $\mathrm{C}_{\max }(\mathbf{b})$ after single doses of ivosidenib (day -3$)$ in patients with cholangiocarcinoma, chondrosarcoma, and other solid tumors (power model with $95 \%$ CI). Point-wise $95 \%$ CIs are shown. Ln-transformed PK parameters were backtransformed to the original scale by exponentiation

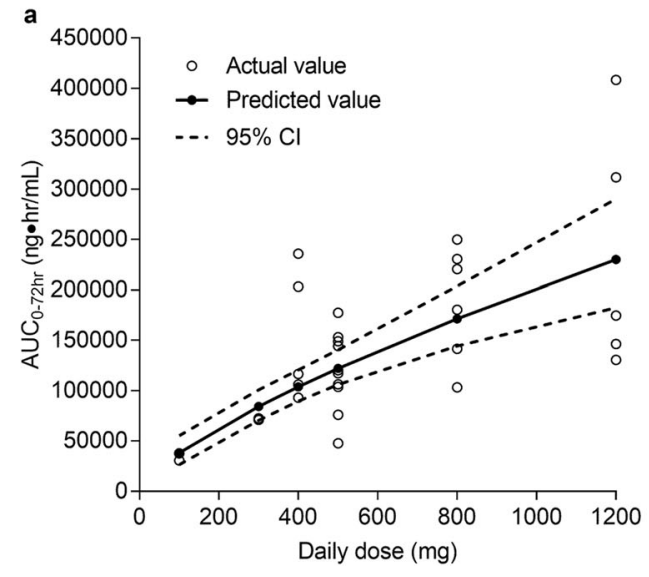

\section{Correlative analysis of ivosidenib PK and PD}

Following single-dose administration of ivosidenib, plasma 2$\mathrm{HG}$ mean $\mathrm{C}_{\mathrm{avg}}$ percent inhibition $\left(\mathrm{BC}_{\mathrm{avg}} \%\right.$ ) was $30 \%$ and $46 \%$ in patients with chondrosarcoma and cholangiocarcinoma, respectively, for ivosidenib $500 \mathrm{mg}$ QD. Plasma 2-HG mean $\mathrm{BC}_{\mathrm{avg}} \%$ was higher following multiple-dose administration of ivosidenib in patients with chondrosarcoma and cholangiocarcinoma for doses of $500 \mathrm{mg}: 54 \%$ and $79 \%$, respectively, at cycle 1 , day 15 , and $59 \%$ and $80 \%$, respectively at cycle 2 , day 1 . With the exception of ivosidenib doses below $500 \mathrm{mg}, 2-$ HG inhibition (as assessed by plasma $\mathrm{AUEC}_{0-8 \mathrm{~h}}$ and $\mathrm{C}_{\mathrm{avg}}$ ) following multiple-dose administration was observed as early as that seen following single-dose administration, with greater 2-HG inhibition after 15 days of QD dosing (cycle 1, day 15). The 2-HG inhibition at cycle 2, day 1 appeared to be similar to cycle 1 , day 15 based on median plasma $\mathrm{C}_{\text {avg }}$ across all dose groups and tumor types (Fig. 3), indicating that steady-state plasma 2-HG inhibition was reached by cycle 1 , day 15 , and was maintained over the course of treatment. There was no additional 2-HG inhibition at doses $>500 \mathrm{mg}$ QD compared with doses of $500 \mathrm{mg}$ QD.

The longitudinal assessment of PK/PD revealed that plasma ivosidenib trough levels were maintained above the predicted efficacious exposure level (determined based on animal PK/PD studies, Agios unpublished data) throughout treatment, and 2-HG inhibition was not only robust but also persistent over the course of treatment. It was also confirmed that the steady state for ivosidenib plasma exposure and plasma 2HG inhibition was reached within 14 days of cycle 1. A representative longitudinal PK/PD plot is shown in Fig. 4 for patients with cholangiocarcinoma receiving $500 \mathrm{mg}$ QD.

Based on data from a limited number of paired tumor biopsy samples (21 patients; all tumor types included), multiple doses of ivosidenib $500 \mathrm{mg}$ QD resulted in substantial 2-HG inhibition of up to $100 \%$ in tumors at cycle 3 , day 1 and cycle 7, day 1 (Supplementary Fig. S3). Based on this limited sample set, exploratory correlations of 2-HG concentration in tumor biopsy samples versus 2-HG concentration in plasma suggested that plasma 2-HG concentration decreased with decreasing $2-\mathrm{HG}$ concentration in tumors.

\section{Correlation of ivosidenib exposure with adverse events and clinical response}

Boxplots of exposure distributions (both $\mathrm{C}_{\mathrm{max}, \mathrm{ss}}$ and $\mathrm{AUC}_{\mathrm{ss}}$ ) in patients who did and did not experience an adverse event in each category outlined in the methods section generally showed the exposure distributions to be similar and overlapping. A representative example of $\mathrm{AUC}_{\mathrm{ss}}$ distribution in patients with or without grade $\geq 3$ adverse events, split by tumor type, is presented in Fig. 5a-b. The observed adverse event incidence by exposure for $\mathrm{AUC}_{\mathrm{ss}}$ or $\mathrm{C}_{\mathrm{max}, \mathrm{ss}}$ did not show clear trends for any of the adverse events assessed. Logistic regression analysis illustrated the absence of the effects of exposure $\left(\mathrm{AUC}_{\mathrm{ss}}\right)$ or $\mathrm{C}_{\mathrm{max}, \mathrm{ss}}$ on the incidence of all selected safety endpoints. Predicted changes over the exposure range were small and not clinically relevant.

Exposure distributions versus best clinical response were also generated. Both $\mathrm{C}_{\mathrm{max}, \mathrm{ss}}$ and $\mathrm{AUC}_{\mathrm{ss}}$ distributions were similar and overlapping among patients with partial response, stable disease and progressive disease (Fig. 5c).

\section{Discussion}

Ivosidenib demonstrated rapid oral absorption and was eliminated slowly, with a long half-life. Ivosidenib exposure increased with increasing dose; however, the increases were less than dose-proportional after both single and multiple doses. After multiple doses (cycle 2, day 1), $\mathrm{AUC}_{0 \text {-tau }}$ and $\mathrm{C}_{\max }$ increased approximately 6-fold and 4-fold, respectively, for a 12-fold increase in dose (100 mg BID to $1200 \mathrm{mg}$ QD across all tumor types). A doubling of dose translates approximately to a $40 \%$ increase in ivosidenib exposure for patients with glioma and a $70 \%$ increase for patients with non-glioma solid 
a

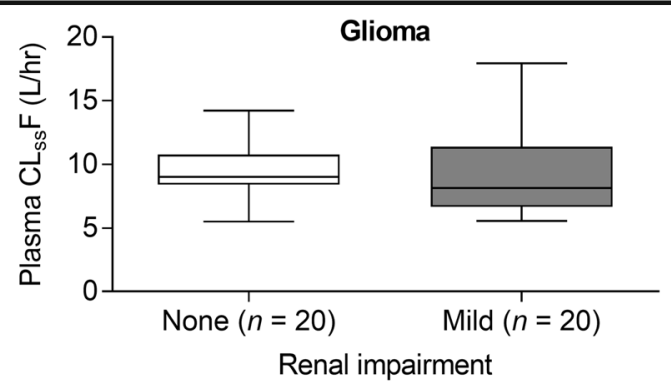

b

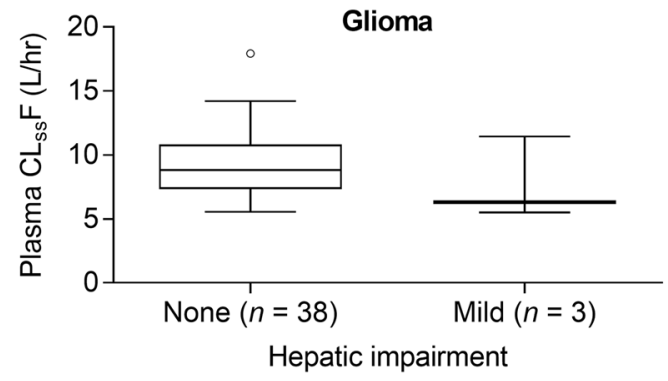

C

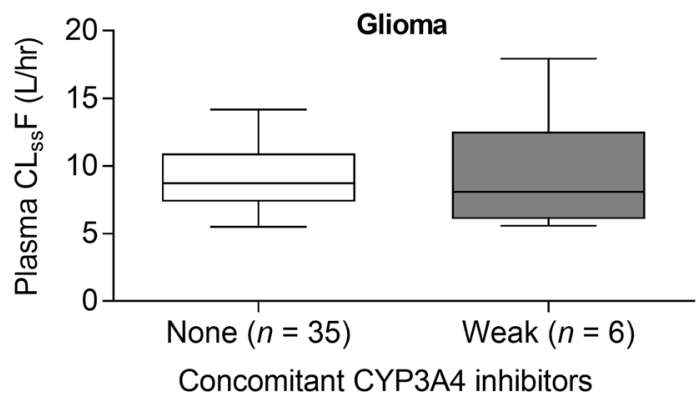

d

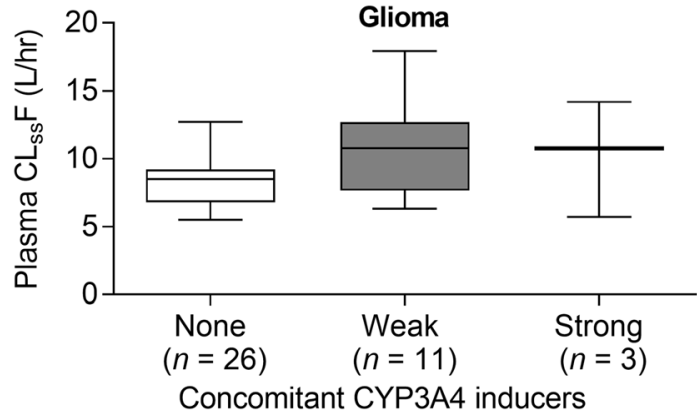

Fig. 2 Plasma ivosidenib CLss/F after multiple oral doses of ivosidenib $500 \mathrm{mg}$ QD (cycle 2, day 1, dose escalation and expansion) by renal function category (based on baseline eGFR) (a), hepatic function category (b), concomitant CYP3A4 inhibitors (c), and concomitant

tumors, across the dose range tested. The solubility, permeability, and dissolution characteristics of ivosidenib have been characterized according to the FDA Biopharmaceutical Classification System (BCS) guidance. Ivosidenib is considered to be a BCS Class II compound (i.e., low solubility, high permeability) (Agios data on file), and hence solubility-

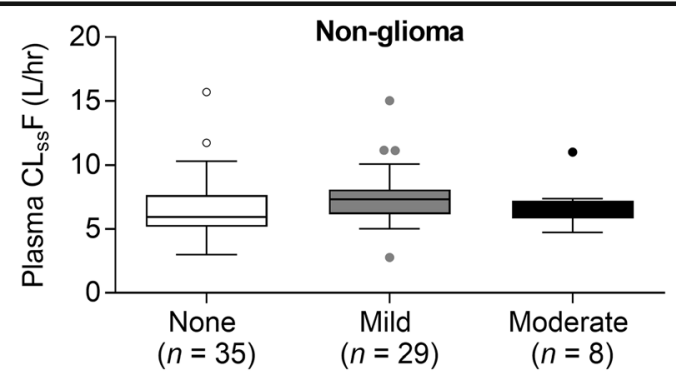

Renal impairment

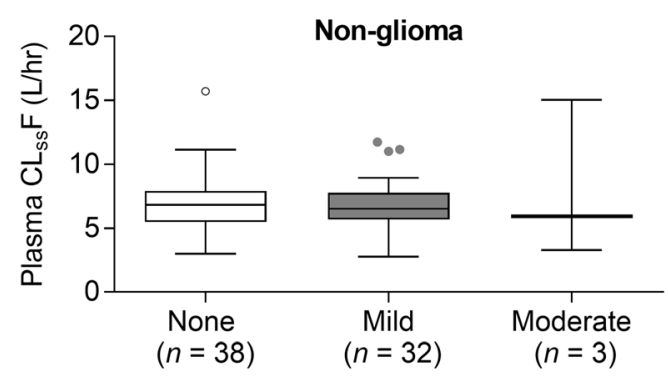

Hepatic impairment

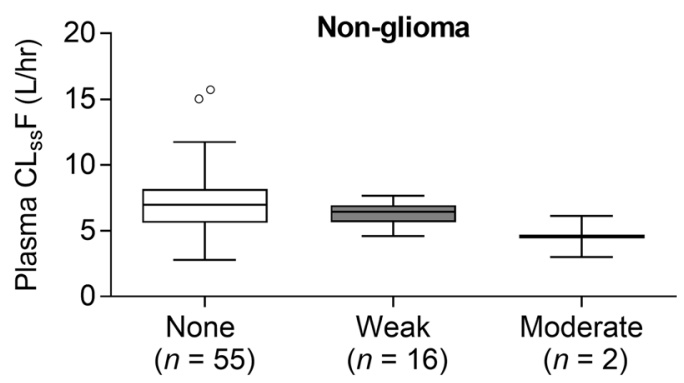

Concomitant CYP3A4 inhibitors

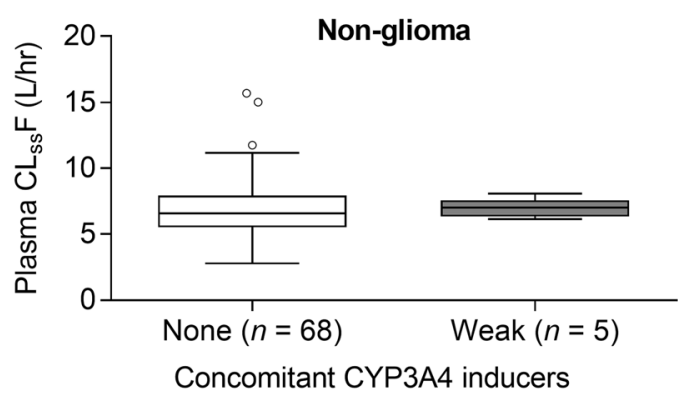

CYP3A4 inducers (d). Glioma includes non-enhancing and enhancing glioma. Non-glioma includes cholangiocarcinoma, chondrosarcoma, and other solid tumors. Horizontal lines denote median; boxes denote 25 th to 75 th percentiles; whiskers were plotted using the Tukey method

limited absorption may contribute to the non-proportionality observed after single and multiple dosing.

The estimated PK parameters for ivosidenib in patients with solid tumors (i.e., cholangiocarcinoma, chondrosarcoma, or glioma) exhibited time-dependency. Based on the mean $t_{1 / 2}$ of $56 \mathrm{~h}$ and $64 \mathrm{~h}$ after a single dose of $500 \mathrm{mg}$ in patients with 
Fig. 3 Summary of average plasma 2-HG concentrations over time by dose category and tumor type (dose escalation and expansion combined) for cholangiocarcinoma (a) and chondrosarcoma (b). The dotted horizontal lines denote average 2HG levels observed in healthy volunteers. Abbreviations: C1D15, cycle 1 day $15 ; \mathrm{C} 2 \mathrm{D} 1$, cycle 2 day 1 . a Cholangiocarcinoma

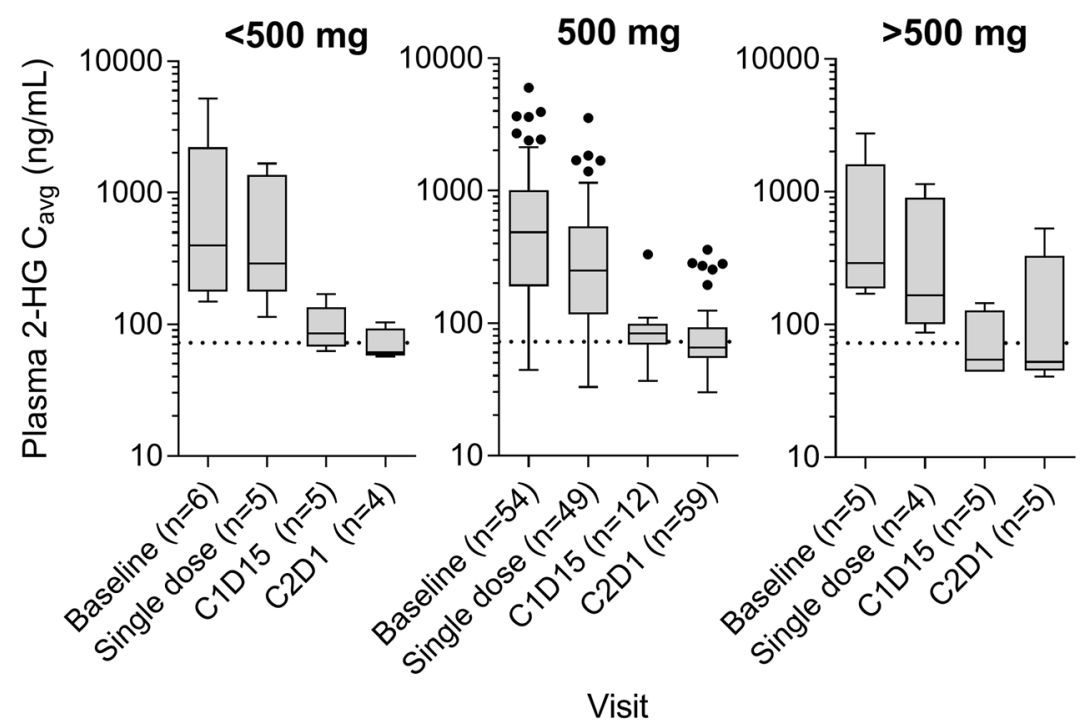

b

Chondrosarcoma

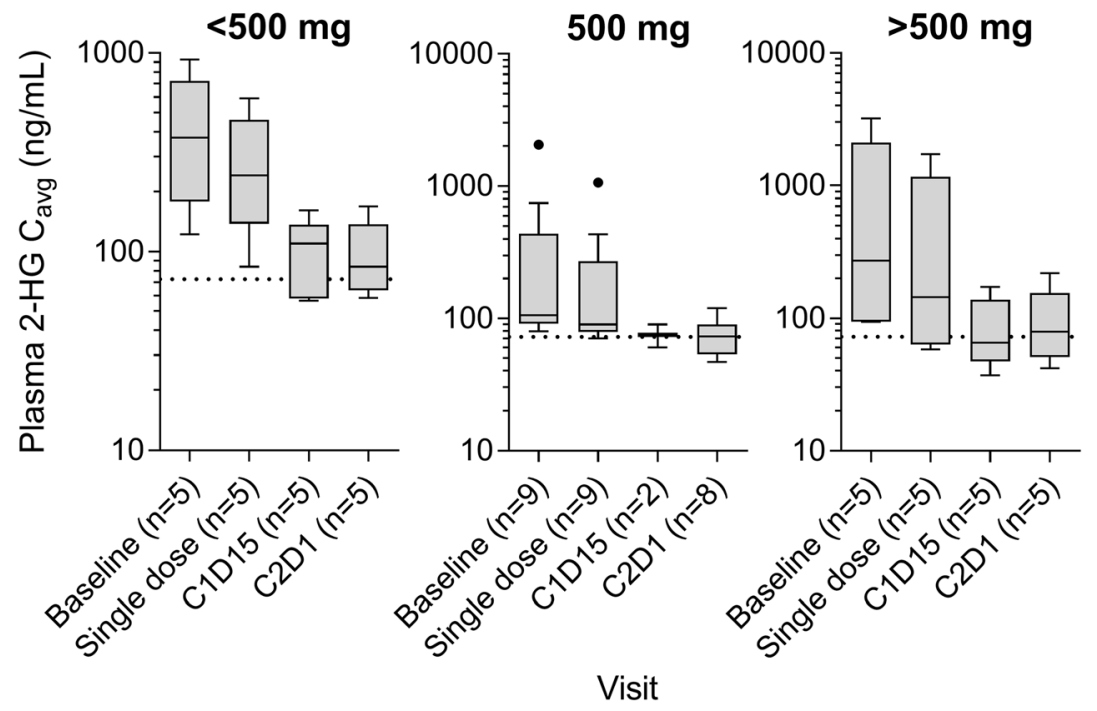

glioma and cholangiocarcinoma/chondrosarcoma, respectively, the anticipated accumulation ratio would be approximately 4-fold after multiple dosing with $500 \mathrm{mg}$ QD, whereas the observed accumulation ratios at cycle 2, day 1 were 1.5 and 1.7 , respectively, suggesting an approximate 2.5 -fold increase in CL/F after multiple dosing. Ivosidenib is mainly metabolized by CYP3A4, and induces CYP3A enzyme activity as suggested by increases in $4 \beta-\mathrm{OHC}$ :cholesterol ratios. On the basis of these data, it is plausible that autoinduction of ivosidenib metabolism may play a role in the observed increase in apparent clearance. However, the magnitude of autoinduction appears to be moderate, and approximately 1.5-fold accumulation was observed at steady state on day 15 .

At the $500 \mathrm{mg}$ QD dose level, $\mathrm{AUC}_{0-24 \mathrm{~h}}$ and $\mathrm{C}_{\max }$ in patients with glioma (enhancing and non-enhancing) were lower than in patients with cholangiocarcinoma/chondrosarcoma. A comparison at the recommended clinical dose of $500 \mathrm{mg}$ QD indicates that the $\mathrm{AUC}_{0-24 \mathrm{~h}}$ and $\mathrm{C}_{\max }$ at steady state of ivosidenib are approximately $33 \%$ and $29 \%$ lower, respectively, in patients with glioma as compared with patients with cholangiocarcinoma/chondrosarcoma. Interindividual variability appears to be lower in patients with glioma than in 
Fig. 4 Longitudinal plots of plasma ivosidenib concentration (a) and percent 2-HG inhibition (b) after oral administration of ivosidenib $500 \mathrm{mg}$ QD in patients with cholangiocarcinoma (dose escalation and expansion combined). The dotted horizontal line in panel a denotes the predicted efficacious level of ivosidenib
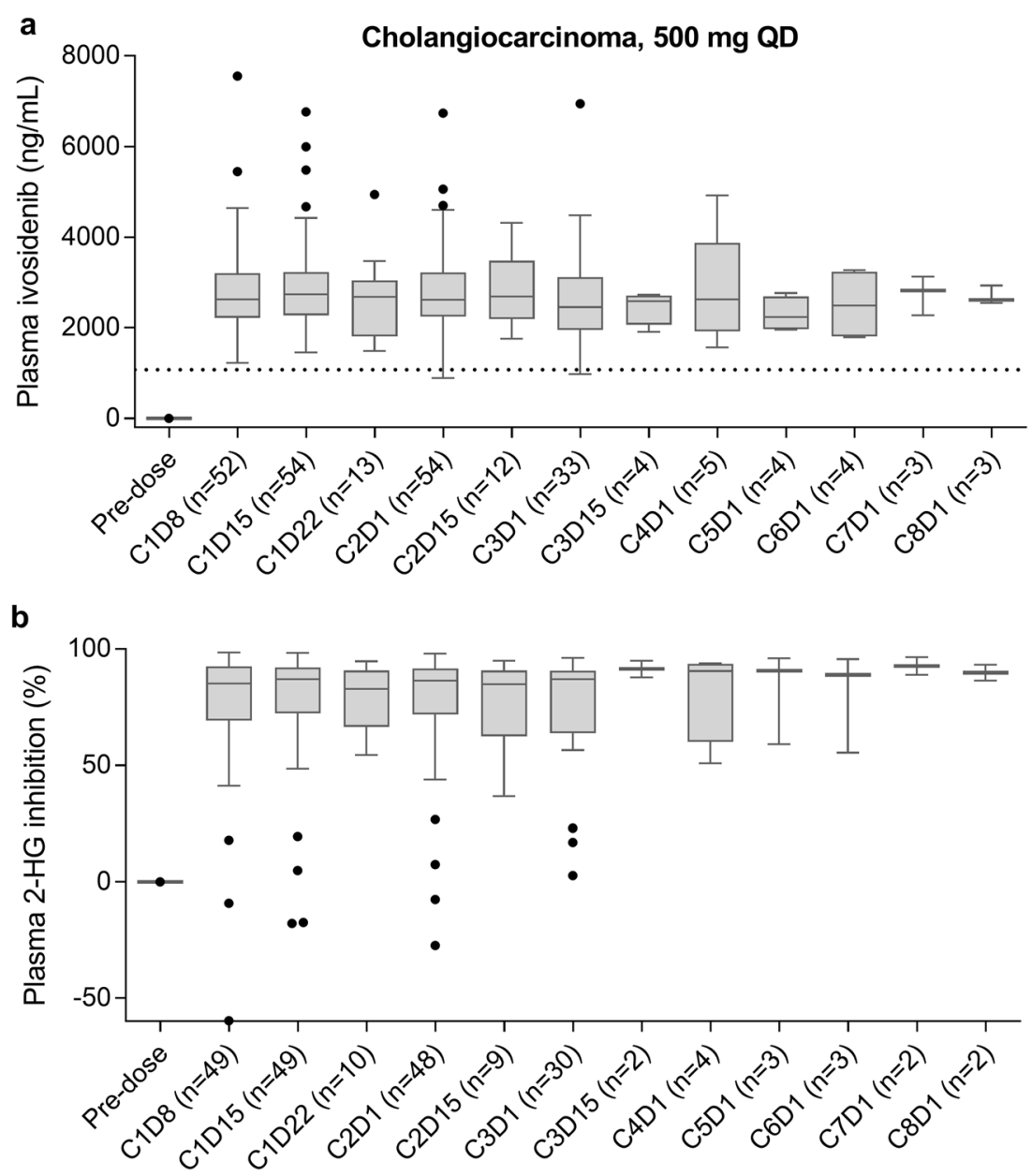

patients with cholangiocarcinoma/chondrosarcoma. The lower exposure in glioma patients may reflect baseline patientspecific factors or disease characteristics in this population, such as lower age, less frequent use of CYP3A4 inhibitors, more frequent use of strong CYP3A4 inducers (e.g., carbamazepine and phenytoin), and adequate renal/hepatic function, as compared with patients with cholangiocarcinoma/ chondrosarcoma.

The determination of the clinical dose of ivosidenib for the expansion portion of the study was based on PD (2-HG inhibition), PK, safety, and efficacy data from the dose escalation portion. Data from the combined dose escalation and expansion portions confirmed that the dose regimen of $500 \mathrm{mg}$ QD ivosidenib appears appropriate for the treatment of patients with advanced solid tumors with an IDH1 mutation.

In patients with cholangiocarcinoma/chondrosarcoma, after multiple doses of ivosidenib, plasma 2-HG levels were substantially reduced (by up to $98 \%$ ), to concentrations similar to those seen in healthy subjects, at all dose levels tested. No additional 2-HG inhibition was observed at doses
$>500 \mathrm{mg}$ QD compared with $500 \mathrm{mg}$ QD, while doses $<500 \mathrm{mg}$ QD appeared to be associated with lower levels of inhibition (although the sample size precluded statistical comparison). Continuous treatment with $500 \mathrm{mg}$ QD was shown to provide persistent 2-HG inhibition, with no decrease in 2HG inhibition over the whole treatment period. Unlike other solid tumor indications, plasma 2-HG does not appear to be a robust PD marker in the glioma population, which may be due to the anatomical location of the tumor. A perioperative study in patients with non-enhancing, IDH1-mutated, low-grade gliomas is being conducted to determine the concentration of ivosidenib and 2-HG in tumors following presurgical treatment with ivosidenib (clinicaltrials.gov NCT03343197). This study is ongoing and aims to confirm the optimal dose of ivosidenib in future glioma studies.

None of the intrinsic patient factors assessed, including renal and hepatic function, had an effect on ivosidenib exposure. Concomitant administration of weak CYP3A4 inhibitors or weak CYP3A4 inducers did not appear to affect the plasma clearance of ivosidenib. These results suggest that dose 
Fig. 5 Exposure-response analyses. Ivosidenib exposure $\left(\mathrm{AUC}_{\mathrm{ss}}\right)$ distribution versus occurrence of grade $\geq 3$ adverse events for patients with cholangiocarcinoma, chondrosarcoma and other solid tumors (a), and glioma (nonenhancing and enhancing) (b). Panel c shows exposure $\left(\mathrm{AUC}_{\mathrm{ss}}\right)$ distribution versus clinical best response in patients with cholangiocarcinoma, chondrosarcoma and other solid tumors. Abbreviations: AE, adverse event; $\mathrm{PD}$, progressive disease; PR, partial response; $\mathrm{SD}$, stable disease

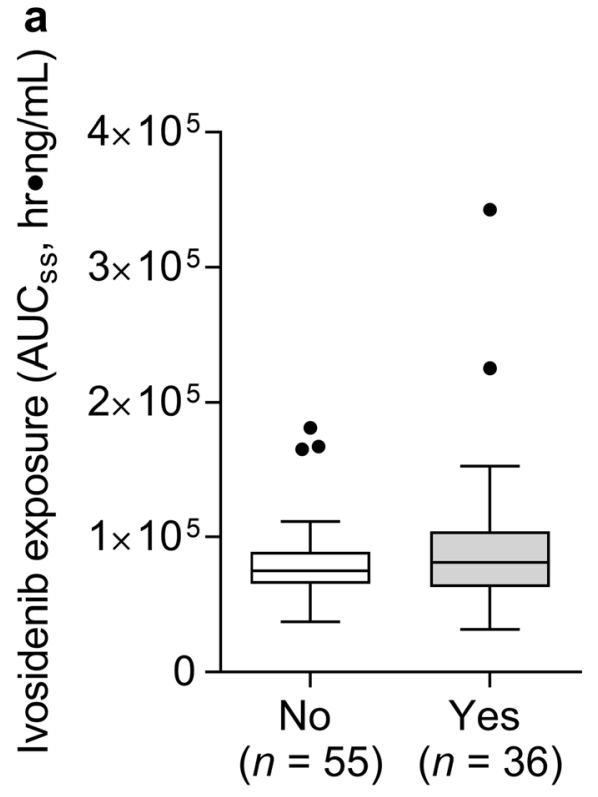

AE grade $>3$ b

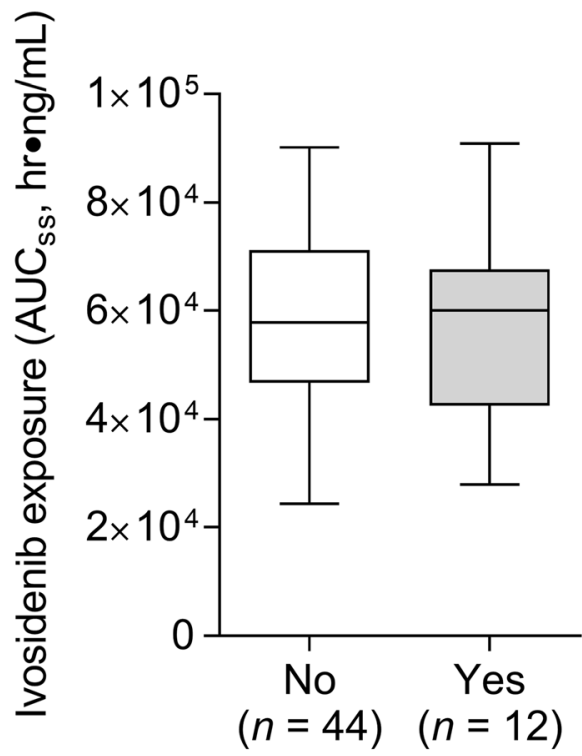

$\mathrm{AE}$ grade $>3$

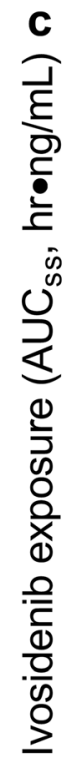

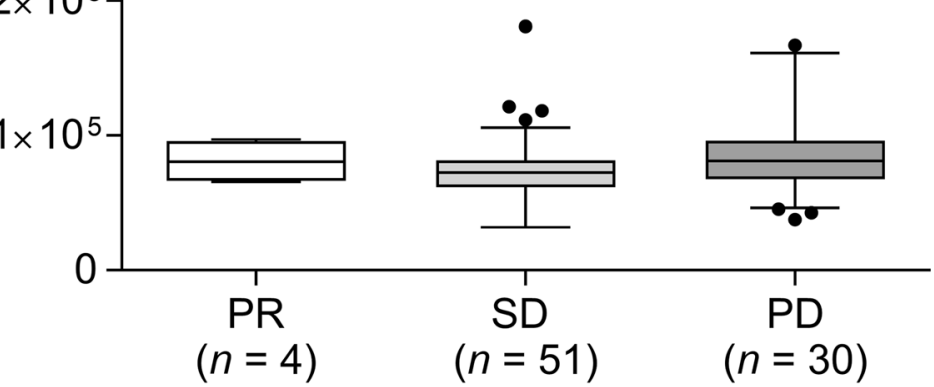

adjustment based on intrinsic patient factors or concomitant use of weak CYP3A4 inhibitors/inducers is not required.

The PK/PD analyses reported here indicate that ivosidenib has flat exposure-safety and exposure-efficacy relationships within the dose range tested. There was no observed effect of exposure on the incidence of any of the adverse events or safety endpoints assessed, and the exposure distribution was similar across all clinical response categories.

As reported elsewhere, ivosidenib has demonstrated encouraging preliminary clinical activity at a dose of $500 \mathrm{mg}$ QD among the cholangiocarcinoma, chondrosarcoma, and non-enhancing glioma populations and is generally well tolerated, with an acceptable toxicity profile and few dose reductions or discontinuations owing to adverse events [11-14]. The data reported here demonstrate that ivosidenib has a favorable PK profile in patients with IDH1-mutated solid tumors, with robust suppression of plasma 2-HG in patients with cholangiocarcinoma or chondrosarcoma. The exposureefficacy and exposure-safety results, combined with the consistency of the clinical benefit observed, suggest that ivosidenib $500 \mathrm{mg}$ QD is an appropriate dose for the treatment of patients with IDH1-mutant advanced solid tumors. 
Acknowledgments The authors would like to thank Certara Strategic Consulting for PK and PD analysis, and Samantha Abel, Valley Writing Solutions Ltd. (Canterbury, UK) and Helen Varley, PhD, CMPP, Excel Medical Affairs (Horsham, UK), for assistance with preparation of the manuscript. This work was funded by Agios Pharmaceuticals, Inc.

Funding This work was funded by Agios Pharmaceuticals, Inc.

\section{Compliance with ethical standards}

Conflict of interest B. Fan, S.S. Pandya, E. Manyak, L. Jiang, G. Liu, T. Nimkar, C. Gliser, M. Prahl Judge, and H. Yang are employees and stockholders of Agios. S. Agresta and D. Dai were employees and stockholders of Agios at the time of the study. E. Manyak was an employee of Agios at the time of the study and current stockholder of Agios. I.K. Mellinghoff has received research funding from General Electric, Amgen, and Lilly; has advisory roles with Agios, Puma Biotechnology, and Debiopharm Group; and has received an honorarium from Roche for a presentation. P.Y. Wen has participated in advisory boards for Abbvie, Agios, AstraZeneca, Blue Earth Diagnostics, Eli Lilly, Genentech/Roche, Immunomic Therapeutics, Kadmon, Kiyatec, Puma, Vascular Biogenics, Taiho, Deciphera, and VBI Vaccines; has acted as speaker for Merck and Prime Oncology; has received research support from Agios, AstraZeneca, Beigene, Eli Lily, Genentech/Roche, Karyopharm, Kazia, MediciNova, Merck, Novartis, Oncoceutics, Sanofi-Aventis, and VBI Vaccines. M.A. Lowery is a consultant and advisory board member for Agios and an advisory board member for Celgene, EMD Serono, and Roche. L. Goyal is an advisory board member for Pieris Pharmaceuticals and Debiopharm. W.D. Tap has received clinical trial funding from Agios during the conduct of the study; personal fees from Eli Lilly, EMD Serono, Novartis, Eisai, Janssen, Immune Design, Adaptimmune, Daiichi Sankyo, Blueprint, Loxo, GlaxoSmithKline, Agios, and Plexxikon; holds a patent for Companion Diagnostic for CDK4 inhibitors - 14/854,329 pending to MSKCC/SKI; a patent for Methods of Treating Metastatic Sarcoma Using Talimogene Laherparepvec (T-Vec) and Pembrolizumab Combination Therapy - 62/671,625 pending to MSKCC/SKI; is a scientific advisory board member and stock owner for Certis Oncology Solutions and Atropos Therapeutics.

Ethical approval All procedures performed in studies involving human participants were in accordance with the ethical standards of the institutional and/or national research committee and with the 1964 Helsinki declaration and its later amendments or comparable ethical standards. This article does not contain any studies with animals performed by any of the authors.

Informed consent Informed consent was obtained from all individual participants included in the study.

Open Access This article is distributed under the terms of the Creative Commons Attribution 4.0 International License (http:// creativecommons.org/licenses/by/4.0/), which permits unrestricted use, distribution, and reproduction in any medium, provided you give appropriate credit to the original author(s) and the source, provide a link to the Creative Commons license, and indicate if changes were made.

\section{References}

1. Dang L, White DW, Gross S, Bennett BD, Bittinger MA, Driggers EM, Fantin VR, Jang HG, Jin S, Keenan MC, Marks KM, Prins RM, Ward PS, Yen KE, Liau LM, Rabinowitz JD, Cantley LC, Thompson CB, Vander Heiden MG, Su SM (2009) Cancer- associated IDH1 mutations produce 2-hydroxyglutarate. Nature 462:739-744. https://doi.org/10.1038/nature08617

2. Ward PS, Patel J, Wise DR, Abdel-Wahab O, Bennett BD, Coller HA, Cross JR, Fantin VR, Hedvat CV, Perl AE, Rabinowitz JD, Carroll M, Su SM, Sharp KA, Levine RL, Thompson CB (2010) The common feature of leukemia-associated IDH1 and IDH2 mutations is a neomorphic enzyme activity converting alphaketoglutarate to 2-hydroxyglutarate. Cancer Cell 17:225-234. https://doi.org/10.1016/j.ccr.2010.01.020

3. Lu C, Ward PS, Kapoor GS, Rohle D, Turcan S, Abdel-Wahab O, Edwards CR, Khanin R, Figueroa ME, Melnick A, Wellen KE, O'Rourke DM, Berger SL, Chan TA, Levine RL, Mellinghoff IK, Thompson CB (2012) IDH mutation impairs histone demethylation and results in a block to cell differentiation. Nature 483:474-478. https://doi.org/10.1038/nature 10860

4. Saha SK, Parachoniak CA, Ghanta KS, Fitamant J, Ross KN, Najem MS, Gurumurthy S, Akbay EA, Sia D, Cornella H, Miltiadous O, Walesky C, Deshpande V, Zhu AX, Hezel AF, Yen KE, Straley KS, Travins J, Popovici-Muller J, Gliser C, Ferrone CR, Apte U, Llovet JM, Wong KK, Ramaswamy S, Bardeesy N (2014) Mutant IDH inhibits HNF- $4 \alpha$ to block hepatocyte differentiation and promote biliary cancer. Nature 513:110-114. https://doi. org/10.1038/nature13441

5. Xu W, Yang H, Liu Y, Yang Y, Wang P, Kim SH, Ito S, Yang C, Wang P, Xiao MT, Liu LX, Jiang WQ, Liu J, Zhang JY, Wang B, Frye S, Zhang Y, Xu YH, Lei QY, Guan KL, Zhao SM, Xiong Y (2011) Oncometabolite 2-hydroxyglutarate is a competitive inhibitor of $\alpha$-ketoglutarate-dependent dioxygenases. Cancer Cell 19:1730. https://doi.org/10.1016/j.ccr.2010.12.014

6. Popovici-Muller J, Lemieux RM, Artin E, Saunders JO, Salituro FG, Travins J, Cianchetta G, Cai Z, Zhou D, Cui D, Chen P, Straley K, Tobin E, Wang F, David MD, Penard-Lacronique V, Quivoron C, Saada V, de Botton S, Gross S, Dang L, Yang H, Utley L, Chen Y, Kim H, Jin S, Gu Z, Yao G, Luo Z, Lv X, Fang C, Yan L, Olaharski A, Silverman L, Biller S, Su SM, Yen K (2018) Discovery of AG-120 (ivosidenib): a first-in-class mutant IDH1 inhibitor for the treatment of IDH1 mutant cancers. ACS Med Chem Lett 9:300-305. https://doi.org/10.1021/acsmedchemlett. $7 \mathrm{~b} 00421$

7. Hansen E, Quivoron C, Straley K, Lemieux RM, Popovici-Muller J, Sadrzadeh H, Fathi AT, Gliser C, David M, Saada V, Micol J-B, Bernard O, Dorsch M, Yang H, Su M, Agresta S, de Botton S, Penard-Lacronique V, Yen K (2014) AG-120, an oral, selective, first-in-class, potent inhibitor of mutant IDH1, reduces intracellular $2 \mathrm{HG}$ and induces cellular differentiation in TF-1 R132H cells and primary human IDH1 mutant AML patient samples treated ex vivo. Blood 124:A3734

8. Fan B, Chen YB, Wang F, Yen K, Utley L, Prahl M, Colby K, Straley K, Bowden C, Biller S, Agresta S, Yang H (2015) Pharmacokinetic/pharmacodynamic evaluation of AG-120, a potent inhibitor of the IDH1 mutant protein, in a phase 1 study of IDH 1-mutant advanced hematologic malignancies. Haematologica 100:218 P572

9. DiNardo CD, Stein EM, de Botton S, Roboz GJ, Altman JK, Mims AS, Swords R, Collins RH, Mannis GN, Pollyea DA, Donnellan W, Fathi AT, Pigneux A, Erba HP, Prince GT, Stein AS, Uy GL, Foran JM, Traer E, Stuart RK, Arellano ML, Slack JL, Sekeres MA, Willekens C, Choe S, Wang H, Zhang V, Yen KE, Kapsalis SM, Yang H, Dai D, Fan B, Goldwasser M, Liu H, Agresta S, Wu B, Attar EC, Tallman MS, Stone RM, Kantarjian HM (2018) Durable remissions with ivosidenib in IDH1-mutated relapsed or refractory AML. N Engl J Med 378:2386-2398. https://doi.org/10.1056/ NEJMoa1716984

10. Agios Pharmaceuticals, Inc. (2018) TIBSOVO $^{\circledR}$ (ivosidenib) prescribing information. https://www.tibsovo.com/pdf/ prescribinginformation.pdf. Accessed 16 Oct 2018 
11. Lowery MA, Abou-Alfa GK, Burris HA, Janku F, Shroff RT, Cleary JM, Azad NS, Goyal L, Maher EA, Gore L, Hollebecque A, Beeram M, Trent JC, Jiang L, Ishii Y, Auer J, Gliser C, Agresta SV, Pandya SS, Zhu AX (2017) Phase 1 study of AG-120, an IDH1 mutant enzyme inhibitor: results from the cholangiocarcinoma dose escalation and expansion cohorts. J Clin Oncol 35:A4015

12. Mellinghoff IK, Touat M, Maher E, de la Fuente M, Cloughesy TF, Holdhoff M, Cote GM, Burris H, Janku F, Huang R, Young RJ, Ellingson B, Nimkar T, Jiang L, Ishii Y, Choe S, Fan B, Steelman L, Yen K, Bowden C, Pandya S, Wen PY (2017) ACTR-46. AG-120, a first-in-class mutant IDH1 inhibitor in patients with recurrent or progressive IDH1 mutant glioma: updated results from the phase 1 non-enhancing glioma population. Neuro-Oncology 19:vi10-vi11

13. Mellinghoff IK, Touat M, Maher E, De La Fuente M, Cloughesy TF, Holdhoff M, Cote G, Burris H, Janku P, Huang R, Young R, Ellingson B, Auer J, Liu H, Hurov J, Yen K, Agresta S, Attar E, Pandya S, Wen PY (2016) ACTR-46. AG-120, a first-in-class mutant IDH1 inhibitor in patients with recurrent or progressive IDH1 mutant glioma: results from the phase 1 glioma expansion cohorts. Neuro-Oncology 18:vi12 ACTR-vi12

14. Tap W, Villalobos VM, Cote GM, Burris H, Janku P, Mir O, Beeram M, Wagner A, Auer J, Liu H, Wu B, Ishii Y, Fan B,
Choe S, Jiang L, Yen K, Agresta S, Pandya SS, Trent J (2016) A phase 1 study of AG-120, an IDH1 mutant enzyme inhibitor: results from the chondrosarcoma dose escalation and expansion cohorts. Poster presented at the Connective Tissue Oncology Society 21st Annual Meeting, Lisbon, Portugal, November 9-12 2016

15. National Kidney Foundation (2002) K/DOQI clinical practice guidelines for chronic kidney disease: evaluation, classification, and stratification. Am J Kidney Dis 39(2 suppl 1):S1-S266

16. Patel H, Egorin MJ, Remick SC, Mulkerin D, Takimoto CHM, Doroshow JH, Potter D, Ivy SP, Murgo AJ, Ramanathan RK (2004) Comparison of Child-Pugh (CP) criteria and NCI organ dysfunction working group (NCI-ODWG) criteria for hepatic dysfunction (HD): implications for chemotherapy dosing. J Clin Oncol 22:A6051

Publisher's note Springer Nature remains neutral with regard to jurisdictional claims in published maps and institutional affiliations.

\section{Affiliations}

Bin Fan ${ }^{1} \cdot$ Ingo K. Mellinghoff ${ }^{2} \cdot$ Patrick Y. Wen $^{3} \cdot$ Maeve A. Lowery $^{4} \cdot$ Lipika Goyal $^{5} \cdot$ William D. Tap $^{6,7}$. Shuchi S. Pandya ${ }^{8} \cdot$ Erika Manyak $^{1} \cdot$ Liewen Jiang $^{9} \cdot$ Guowen Liu $^{1} \cdot$ Tara Nimkar $^{10} \cdot$ Camelia Gliser $^{8} \cdot$ Molly Prahl Judge $^{8} \cdot$ Sam Agresta $^{8} \cdot$ Hua Yang $^{1} \cdot$ David Dai $^{1}$

$1 \quad$ DMPK, Agios Pharmaceuticals, Inc., 88 Sidney Street, Cambridge, MA 02139, USA

2 Departments of Neurology and Human Oncology and Pathogenesis Program, Memorial Sloan Kettering Cancer Center, New York, NY, USA

3 Center for Neuro-Oncology, Dana-Farber Cancer Institute, Boston, MA, USA

4 Trinity St James Cancer Institute, Trinity College, Dublin, Ireland

5 Department of Internal Medicine, Division of Hematology/ Oncology, Massachusetts General Hospital Cancer Center, Boston, MA, USA
6 Department of Medicine, Memorial Sloan Kettering Cancer Center, New York, NY, USA

7 Department of Medicine, Weill Cornell Medical College, New York, NY, USA

8 Clinical Development, Agios Pharmaceuticals, Inc., Cambridge, MA, USA

9 Biostatistics - Clinical Development, Agios Pharmaceuticals, Inc., Cambridge, MA, USA

10 Clinical Operations - Clinical Development, Agios Pharmaceuticals, Inc., Cambridge, MA, USA 